\title{
Seroepidemiology of Helicobacter pylori infection in general population in a northern Mexican city
}

\author{
Cosme Alvarado-Esquivel ${ }^{1^{*}}$, Agar Ramos-Nevárez ${ }^{2}$, Sandra Margarita Cerrillo-Soto², \\ Lucio Martínez-Ramírez ${ }^{2}$, Carlos Alberto Guido-Arreola², Fernando Arturo Muñoz-González ${ }^{2}$ \\ ${ }^{1}$ Biomedical Research Laboratory, Faculty of Medicine and Nutrition, Juárez University of Durango State, Durango, Mexico; \\ *Corresponding Author: alvaradocosme@yahoo.com \\ ${ }^{2}$ Clínica de Medicina Familiar, Instituto de Seguridad y Servicios Sociales de los Trabajadores del Estado (ISSSTE), Durango, \\ México
}

Received 19 November 2013; revised 21 December 2013; accepted 29 December 2013

Copyright (C 2014 Cosme Alvarado-Esquivel et al. This is an open access article distributed under the Creative Commons Attribution License, which permits unrestricted use, distribution, and reproduction in any medium, provided the original work is properly cited. In accordance of the Creative Commons Attribution License all Copyrights (C) 2014 are reserved for SCIRP and the owner of the intellectual property Cosme Alvarado-Esquivel et al. All Copyright (C) 2014 are guarded by law and by SCIRP as a guardian.

\section{ABSTRACT}

Background: The seroepidemiology of Helicobacter pylori infection in general population in Durango City, Mexico has not been previously studied. Aims: To determine the seroprevalence of anti-H. pylori antibodies in the general population in Durango City, Mexico; and to determine socio-demographic, clinical, and behavioral characteristics of the general population associated with $H$. pylori seropositivity. Methods: The prevalence of anti-H. pylori IgG antibodies was examined in 345 individuals in Durango City, Mexico, using enzyme-linked immunoassays. Sociodemographic, clinical, and behavioral characteristics of the general population associated with $H$. pylori seroprevalence were also investigated. Results: In total, 231 (67\%) of the 345 participants (mean age, 45.3. \pm 17.5 years) had $H$. pylori IgG antibodies, 146 (63.2\%) of whom had $H$. pylori IgG antibody levels higher than $100 \mathrm{U} / \mathrm{mL}$. Both $\boldsymbol{H}$. pylori seroprevalence and antibody levels increased significantly with age, and were similar in males and females. An increased $\boldsymbol{H}$. pylori seroprevalence was found in subjects with low education and employed. In contrast, a low seroprevalence was found in subjects living in suburban areas. One of four subjects with gastritis symptoms had anti-H. pylori antibodies. The seroprevalence of $\boldsymbol{H}$. pylori was significantly higher among women with abortion history than among those without such history. Multivariate analysis of behavioral variables showed that $\boldsymbol{H}$. pylori seropositivity was negatively associated with raising farm animals $(\mathrm{OR}=0.48 ; 95 \% \mathrm{Cl}$ : $0.25-0.91 ; P=0.02$ ). Conclusions: In the general population studied, $\boldsymbol{H}$. pylori seroreactivity increased significantly with age, not only in frequency but also in intensity. Residence, education, and occupation of the study population influenced the seropositivity rate of $\boldsymbol{H}$. pylori.

\section{KEYWORDS}

Helicobacter pylori; Seroprevalence; General Population; Epidemiology; Mexico

\section{INTRODUCTION}

Helicobacter pylori is a widely distributed pathogen causing infections in humans worldwide [1]. Estimates indicate that about one-half of the world's population is currently infected with $H$. pylori [1,2]. Infections with $H$. pylori may lead to severe gastric diseases including chronic gastritis, peptic ulcer, gastric mucosa-associated lymphoid tissue lymphoma, and gastric cancer [1-4]. Furthermore, increasing evidence suggests that $H$. pylori infection may lead to extra gastric diseases including cardiovascular and neurological diseases, hematologic disorders and others $[5,6]$. Therefore, exploring new clinical manifestations associated with $H$. pylori infection is justified. The routes of transmission of $H$. pylori to humans are not fully understood. However, putative routes of $\mathrm{H}$. pylori dissemination include person-to-person [7], oral-oral or fecaloral [8], and consumption of contaminated water [8,9]. The prevalence of $H$. pylori infection varies substantially among countries and is higher in developing than in developed countries [10]. The seroprevalence of $H$. pylori 
infection may be influenced by a number of factors including geographical region, migration pattern, ethnic group [8,11], and lifestyle [12]. Therefore, exploring the association of $H$. pylori infection with new factors might be useful for a better understanding of the $H$. pylori dissemination.

Very little is known about the seroepidemiology of $H$. pylori infection in Mexico in general and in the northern Mexican state of Durango in particular. In the present survey, the seroprevalence of anti- $H$. pylori antibodies in the general population in Durango City, Mexico was examined. In addition, this study was aimed to determine socio-demographic, clinical, and behavioral characteristics of the general population associated with $H$. pylori seropositivity.

\section{METHODS}

Through a cross-sectional survey, 345 individuals of the general population living in Durango City, Mexico were studied. Inclusion criteria for voluntary participation of the subjects were individuals living in Durango City of 16 years of age and older. Gender, occupation or socio-economic status were not restrictive criteria for enrollment.

Characteristics of the subjects were examined using a standardized questionnaire. Socio-demographic data examined included age, gender, birthplace, residence, educational level, occupation, and socioeconomic status. Clinical data including the presence of: any diseases, history of lymphadenopathy, gastritis, peptic ulcer, and blood transfusion was also obtained from the participants. Obstetric records of women were obtained. Behavioral data examined included animal contact, foreign travel, frequency of eating away from home (in restaurants or fast food outlets), contact with soil (gardening or agriculture), type of flooring at home, type of meat consumed, frequency of meat consumption, degree of meat cooking, consumption of unpasteurized milk, unwashed raw vegetables or fruits, untreated water, or alcohol, and smoking.

Whole blood from the participants was centrifuged and serum samples were kept frozen at $-20^{\circ} \mathrm{C}$ until analyzed. Serum samples were examined by qualitative and quantitative methods for detection of anti- $H$. pylori IgG antibodies using a commercially available enzyme-linked immunosorbent assay (ELISA) kit, Anti-H. pylori IgG AccuBind ELISA (Monobind Inc, Lake Forest, California). Anti-H. pylori IgG antibody levels were expressed as Units $(\mathrm{U}) / \mathrm{mL}$, and a value higher than $20 \mathrm{U} / \mathrm{mL}$ was considered a positive result. All tests were performed following the instructions of the manufacturer.

Statistical analysis was performed with the aid of the Epi Info version 3.5.4 software and SPSS version 15.0 software. For calculation of the sample size, a reference seroprevalence of $66 \%$ [13] as the expected frequency for the factor under study, 550,000 as the population size from which the sample was selected, $61 \%$ as the least acceptable result, and a 95\% confidence level were considered. The result of the sample size calculation was 345 subjects. Frequencies between groups were compared with the Pearson's chi-square test and the Fisher exact test (when values were less than 5). Bivariate and multivariate analyses were used to assess the association between $H$. pylori seropositivity and subject characteristics. Variables were included in the multivariate analysis if they had a $P$ value $\leq 0.20$ in the bivariate analysis. Odd ratio (OR) and 95\% confidence interval (CI) were calculated by multivariate analysis, using the Enter method. A $P$ value $<0.05$ was considered statistically significant.

This study was approved by the Ethical Committee of the Instituto de Seguridad y Servicios Sociales de los Trabajadores del Estado in Durango City. Mexico. The purpose and procedures of the study were explained to all participants. In addition, a written informed consent was obtained from all participants and from the next of kin of minor participants.

\section{RESULTS}

H. pylori IgG antibodies were detected in 231 (67\%) of the 345 subjects studied. A correlation between the seroprevalence of $H$. pylori infection and a selection of socio-demographic and behavioral characteristics of the subjects is shown in Table 1 . The majority of participants were born in Durango State; their mean age was $45.3 \pm 17.5$ years (range, 16 - 91 years). Males and females had comparable rates of $H$. pylori seroprevalence (70.8\% and $64.4 \%$, respectively; $P=0.21$ ). However, $H$. pylori seroprevalence rates increased significantly with age $(P<0.001)$. An increased seroprevalence was found in subjects with low education and employed. In contrast, a low seroprevalence was found in subjects living in suburban areas. Other socio-demographic characteristics such as origin and socio-economic status did not significantly influence the seroprevalence of $H$. pylori. Of the $231 \mathrm{H}$. pylori IgG positive participants, 145 (62.8\%) had IgG levels higher than $100 \mathrm{U} / \mathrm{mL}, 41$ (17.7\%) between 51 to $100 \mathrm{U} / \mathrm{mL}$, and 45 (19.5\%) between 21 to $50 \mathrm{U} / \mathrm{mL}$. Anti-H. pylori IgG antibody levels were similar in men and women $(P=0.56)$. In contrast, the frequency of high $(>100 \mathrm{U} / \mathrm{mL})$ anti-H. pylori IgG antibody levels increased significantly with age: $20.5 \%$ in the $\leq 30$ years old group, $44.5 \%$ in the 31 - 50 years old group, and $53 \%$ in the $>50$ years old group $(P=0.00001)$.

Bivariate analysis of behavioral characteristics revealed six variables with a $P$ value $\leq 0.20$ : cats at home $(P=$ $0.18)$, dogs at home $(P=0.10)$, raising farm animals $(P<$ $0.0001)$, consumption of untreated water $(P=0.08)$, eating away of home $(P=0.02)$, and smoking $(P=0.06)$. 
Table 1. Bivariate analysis of selected exposure variables and seroprevalence of $H$. pylori infection in general population.

\begin{tabular}{|c|c|c|c|c|c|c|}
\hline \multirow[t]{2}{*}{ Characteristic } & \multirow{2}{*}{$\begin{array}{c}\text { No. of } \\
\text { Subjects } \\
\text { Tested }^{\mathrm{a}}\end{array}$} & \multicolumn{2}{|c|}{$\begin{array}{c}\text { Positive } \\
\text { ELISA results }\end{array}$} & \multirow{2}{*}{$\begin{array}{l}\text { Odds } \\
\text { Ratio }\end{array}$} & \multirow{2}{*}{$\begin{array}{c}95 \% \\
\text { Confidence } \\
\text { Interval }\end{array}$} & \multirow{2}{*}{$\begin{array}{c}P \\
\text { value }\end{array}$} \\
\hline & & No. & $\%$ & & & \\
\hline \multicolumn{7}{|l|}{ Gender } \\
\hline Male & 137 & 97 & 70.8 & 1.3 & $0.82-2.19$ & 0.21 \\
\hline Female & 208 & 134 & 64.4 & 1.0 & & \\
\hline \multicolumn{7}{|l|}{ Age (years) } \\
\hline 30 or less & 83 & 35 & 42.2 & 1.0 & & \\
\hline $31-50$ & 128 & 87 & 68.0 & 2.9 & $1.58-5.38$ & 0.0002 \\
\hline$>50$ & 134 & 109 & 81.3 & 6.0 & $3.09-11.63$ & $<0.0001$ \\
\hline \multicolumn{7}{|l|}{ Birth place } \\
\hline Durango State & 299 & 205 & 68.6 & 1.0 & & \\
\hline Other State & 40 & 23 & 57.5 & 0.6 & $0.30-1.28$ & 0.16 \\
\hline \multicolumn{7}{|l|}{ Residence place } \\
\hline Durango State & 341 & 231 & 67.7 & 1.0 & & \\
\hline Other State & 4 & 0 & 0.0 & 0.0 & $0.00-0.74$ & 0.01 \\
\hline \multicolumn{7}{|l|}{ Residence area } \\
\hline Urban & 311 & 212 & 68.2 & 1.0 & & \\
\hline Suburban & 15 & 6 & 40.0 & 0.3 & $0.09-1.01$ & 0.02 \\
\hline Rural & 18 & 12 & 66.7 & 0.9 & $0.31-2.89$ & 0.89 \\
\hline \multicolumn{7}{|l|}{ Educational level } \\
\hline Up to 6 years & 45 & 36 & 80.0 & 2.4 & $1.03-5.69$ & 0.02 \\
\hline $7-12$ & 116 & 81 & 69.8 & 1.4 & $0.82-2.34$ & 0.2 \\
\hline$>12$ & 182 & 114 & 62.6 & 1.0 & & \\
\hline \multicolumn{7}{|l|}{ Occupation } \\
\hline Employed $^{\mathrm{b}}$ & 247 & 176 & 71.3 & 2.1 & $1.24-3.52$ & 0.002 \\
\hline Unemployed $^{\mathrm{c}}$ & 94 & 51 & 54.3 & 1.0 & & \\
\hline \multicolumn{7}{|l|}{ Cats at home } \\
\hline Yes & 102 & 63 & 61.8 & 0.7 & $0.43-1.20$ & 0.18 \\
\hline No & 243 & 168 & 69.1 & 1.0 & & \\
\hline \multicolumn{7}{|l|}{ Dogs at home } \\
\hline Yes & 244 & 157 & 64.3 & 0.7 & $0.38-1.13$ & 0.1 \\
\hline No & 101 & 74 & 73.3 & 1.0 & & \\
\hline \multicolumn{7}{|c|}{ Raising farm animals } \\
\hline Yes & 131 & 71 & 54.2 & 0.4 & $0.24-0.64$ & $<0.0001$ \\
\hline No & 213 & 160 & 75.1 & 1.0 & & \\
\hline \multicolumn{7}{|l|}{ Untreated water } \\
\hline Yes & 135 & 83 & 61.5 & 0.7 & $0.41-1.08$ & 0.08 \\
\hline No & 210 & 148 & 70.5 & 1.0 & & \\
\hline \multicolumn{7}{|l|}{ Eating out of home } \\
\hline$\leq 10$ times/yr & 151 & 111 & 73.5 & 1.7 & $1.06-2.82$ & 0.02 \\
\hline$>10$ times/yr & 193 & 119 & 61.7 & 1.0 & & \\
\hline \multicolumn{7}{|l|}{ Smoking } \\
\hline Yes & 41 & 32 & 78 & 2.1 & $0.90-4.93$ & 0.06 \\
\hline No & 228 & 144 & 63.2 & 1.0 & & \\
\hline
\end{tabular}

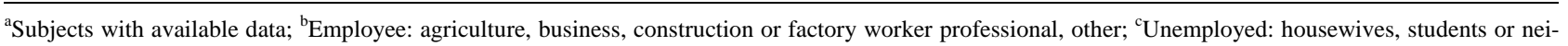
ther. 
Other behavioral characteristics had $P$ values $>0.20$ in the bivariate analysis including foreign travel, contact with soil, type of flooring at home, type of meat consumption, frequency of meat consumption, degree of meat cooking, consumption of unpasteurized milk, unwashed raw vegetables and fruits, and alcohol. Multivariate analysis of behavioral variables with a $P$ value $\leq 0.20$ showed that $H$. pylori seropositivity was only negatively associated with raising farm animals (OR $=0.48$; $95 \% \mathrm{CI}$ : 0.25 - 0.91; $P=0.02$ ) (Table 2).

Analysis of clinical data showed that $H$. pylori positive and $H$. pylori negative participants had similar frequencies of underlying diseases, lymphadenopathy and blood transfusions. Four subjects suffered from gastritis and one of them was positive for anti-H. pylori IgG antibodies. None of the participants suffered from peptic ulcer. Of the obstetric characteristics of women, the seroprevalence of $H$. pylori was significantly $(P=0.04)$ higher among women with abortion history (29/34; 85.3\%) than among those without such history (72/106; 67.9\%). Stratification by number of abortions did not show statistically significant differences in the seroprevalences in women without abortion and those with one (22/26: $84.6 \% ; P=0.09$ ) or between 2 to 5 abortions (7/8: 87.5\%; $P=0.23$ ). The seroprevalence of $H$. pylori exposure was similar between women who had had pregnancies (93/ 130; 71.5\%), deliveries (77/108; 71.3\%), and cesarean sections (31/44; 70.5\%), than women without such obstetric characteristics $(8 / 11 ; 72.7 \%, 24 / 33 ; 72.7 \%$ and 70/96; 72.9\%; $P=0.61$. $P=0.87$ and $P=0.76$, respectively).

\section{DISCUSSION}

The $67 \%$ seroprevalence of $H$. pylori infection in the general population of Durango City found in the present study is similar to the mean national seroprevalence (66\%) reported in another study in Mexico [13]. However, these figures cannot be directly compared since different methods were used in both studies; although this study used a commercial ELISA kit, a homemade ELISA kit was used for the national study. The national study [13] did not provide a specific seroprevalence for Durango. To

Table 2. Results of the multivariate regression analysis.

\begin{tabular}{ccccc}
\hline Variable & $\begin{array}{c}\text { Regression } \\
\text { coefficient }\end{array}$ & $\begin{array}{c}P \\
\text { value }\end{array}$ & $\begin{array}{c}\text { Odds } \\
\text { ratio }\end{array}$ & $\begin{array}{c}95 \% \text { confidence } \\
\text { interval }\end{array}$ \\
\hline Cats at home & 0.09 & 0.77 & 1.1 & $0.56-2.13$ \\
Dogs at home & -0.39 & 0.24 & 0.67 & $0.34-1.31$ \\
Raising farm animals & -0.73 & 0.02 & 0.48 & $0.25-0.91$ \\
Untreated water & -0.21 & 0.49 & 0.8 & $0.42-1.51$ \\
$\begin{array}{c}\text { Low frequency } \\
\text { of eating out of home }\end{array}$ & 0.33 & 0.24 & 1.39 & $0.79-2.44$ \\
Smoking & 0.39 & 0.34 & 1.48 & $0.65-3.38$ \\
\hline
\end{tabular}

the best of our knowledge, the present study is the first one conducted in general population in Durango City. In a regional context, two previous surveys of $H$. pylori infection have been performed, and both of them are in ethnic groups (Mennonites and Tepehuanos) in Durango State. In Mennonites, a 50.7\% seroprevalence of $H$. pylori infection was reported [14]. This seroprevalence is lower than the one found in the present study. The difference in the seroprevalence between the two populations might be due to differences in the general characteristics of the subjects studied. The general population in Durango City is urban while Mennonites are rural. On the other hand, the seroprevalence found in the present study is similar to the $66 \%$ seroprevalence of $\mathrm{H}$. pylori infection found in Tepehuanos [15]. In both studies in Mennonites and Tepehuanos, the same commercial ELISA kit was used. In an international context, the seroprevalence of $H$. pylori infection in the general population of Durango is lower than the estimated $80 \%$ to $90 \%$ seroprevalence of $H$. pylori infection in developing countries [10].

With respect to socio-demographic characteristics, a number of variables correlated with $H$. pylori infection. Firstly, $H$. pylori seroprevalence increased significantly with age. This finding is consistent with the typical increase in the frequency of infection with age reported elsewhere $[7,11,16]$. Secondly, subjects with low education and employed had an increased seroprevalence of $H$. pylori infection. A low educational level has been reported as a factor associated with $H$. pylori infection [13]. However, it is not clear why employed subjects had a higher seroprevalence of $H$. pylori infection than those without employment. In a previous study in Tepehuanos in Durango, $H$. pylori infection was similarly associated with a laborer occupation [15]. It is likely that work factors might have contributed to infection. Although work factors were not investigated in this study, other researchers have found a number of work factors associated with $H$. pylori infection including deployment in a crowded commission combined with the absence of air conditioning in military personnel sleeping quarters [17]. Therefore, studying work factors including crowding, presence of air conditioning, and other factors would be useful for determining their role in $H$. pylori infection. Thirdly, subjects living in suburban areas had a low seroprevalence of $H$. pylori infection. Interpretation of such finding should be interpreted with care since a low number of subjects living in suburban areas were enrolled in the study. The low seroprevalence in subjects living in suburban areas found in the present study conflicts with previous observations of an association of $H$. pylori infection with living in a suburban area in studies in Turkey [18] and Nepal [19]. Differences in the associations might be due to differences in socio-demographic and 
behavioral characteristics among the populations. Socioeconomic status has been linked to H. pylori seropositivity $[7,13]$. However, in the current study no association between $H$. pylori seropositivity and socioeconomic status was found. Remarkably, in the present study the frequency of high anti- $H$. pylori IgG antibody levels increased significantly with age. To the best of our knowledge, such association in general population has not been reported. This finding raises the question whether older subjects are not only more exposed to $H$. pylori infection but also with more intensity than younger subjects are. Further studies should address this issue.

Concerning behavioral characteristics, logistic regression showed that $H$. pylori seropositivity was only negatively associated with raising farm animals. This finding might reflect a rural origin of some seronegative subjects. The seroprevalence of $H$. pylori infection has been found lower in subjects living in rural areas than those living in urban areas [20]. The variable rural residence was not associated with infection in the current study, and other unknown factors may have contributed to infection. It is possible that crowding, a putative risk factor for $\mathrm{H}$. pylo$r i$ infection [17] could be less intense in subjects who raise farm animals since such occupation is done in open areas in the countryside. Further research to clarify the negative association of raising farm animals with $H$. pylori infection is needed.

Concerning clinical data, the seroprevalence of $H$. pylori was significantly higher among women with abortion history than among those without such history. In a previous study in the ethnic group of Mennonites in Durango, Mexico, a similar association between abortion history and $H$. pylori seropositivity was found [14]. In the present study, the mean age in women with abortion history (52.20 \pm 13.60 years old) was comparable with that in women without such obstetric characteristic (47.29 \pm 16.68 years old $)(P=0.12)$. This was the case in the present study as well as in the previous study in Mennonites [14]. In a recent study in the ethnic group of Tepehuanos in the region, no association between abortion history and $H$. pylori seropositivity was found [15]. The finding of the association of $H$. pylori seropositivity with abortion history deserves further research.

\section{ACKNOWLEDGEMENTS}

The authors thank Fidela Rosas-Meza for her valuable support in submitting the questionnaires.

\section{REFERENCES}

[1] Ruggiero, P. (2010) Helicobacter pylori and inflammation. Current Pharmaceutical Design, 16, 4225-4236. http://dx.doi.org/10.2174/138161210794519075

[2] Sachs, G., Scott, D.R. and Wen, Y. (2011) Gastric infec- tion by Helicobacter pylori. Current Gastroenterology Reports, 13, 540-546.

http://dx.doi.org/10.1007/s11894-011-0226-4

[3] Malfertheiner, P. (2011) The intriguing relationship of Helicobacter pylori infection and acid secretion in peptic ulcer disease and gastric cancer. Digestive Diseases, 29, 459-464. http://dx.doi.org/10.1159/000332213

[4] Zabaleta, J. (2012) Multifactorial etiology of gastric cancer. Methods in Molecular Biology, 863, 411-435. http://dx.doi.org/10.1007/978-1-61779-612-8 26

[5] Andrabi, S.A., Shamila, H. and Masooda, S. (2012) Advancement of scientific research on Helicobacter pylori in humans: Where do we stand? East African Journal of Public Health, 9, 118-122.

[6] Banić, M., Franceschi, F., Babić, Z. and Gasbarrini, A. (2012) Extragastric manifestations of Helicobacter pylori infection. Helicobacter, 17, 49-55.

http://dx.doi.org/10.1111/j.1523-5378.2012.00983.x

[7] Ford, A.C. and Axon, A.T. (2010) Epidemiology of Helicobacter pylori infection and public health implications. Helicobacter, 15, 1-6. http://dx.doi.org/10.1111/j.1523-5378.2010.00779.x

[8] Goh, K.L., Chan, W.K., Shiota, S. and Yamaoka, Y. (2011) Epidemiology of Helicobacter pylori infection and public health implications. Helicobacter, 16, 1-9.

http://dx.doi.org/10.1111/j.1523-5378.2011.00874.x

[9] Fedichkina, T.P. and Solenova, L.G. (2011) Helicobacter pylori: Routes of transmission of infection (a review of literature). Gigiena i Sanitariia, 4, 30-34.

[10] Bures, J., Kopácová, M., Skodová Fendrichová, M. and Rejchrt, S. (2011) Epidemiology of Helicobacter pylori infection. Vnitrní Lékarství, 57, 993-999.

[11] Muhsen, K., Cohen, D., Spungin-Bialik, A. and Shohat, T. (2012) Seroprevalence, correlates and trends of Helicobacter pylori infection in the Israeli population. Epidemiology and Infection, 140, 1207-1214. http://dx.doi.org/10.1017/S0950268811002081

[12] Naja, F., Kreiger, N. and Sullivan, T. (2007) Helicobacter pylori infection in Ontario: Prevalence and risk factors. Canadian Journal of Gastroenterology, 21, 501-506.

[13] Torres, J., Leal-Herrera, Y., Perez-Perez, G., Gomez, A., Camorlinga-Ponce, M., Cedillo-Rivera, R., Tapia-Conyer, R. and Muñoz, O. (1998) A community-based seroepidemiologic study of Helicobacter pylori infection in Mexico. Journal of Infectious Diseases, 178, 1089-1094. http://dx.doi.org/10.1086/515663

[14] Alvarado-Esquivel, C. (2012) Seroepidemiology of Helicobacter pylori Infection in a Mennonite Community in Durango State, Mexico. Helicobacter, 18, 215-221. http://dx.doi.org/10.1111/hel.12032

[15] Alvarado-Esquivel, C. (2013) Seroepidemiology of Helicobacter pylori infection in Tepehuanos aged 15 years and older in Durango, Mexico. Journal of Pathogens, 2013, Article ID: 243246.

[16] Sasidharan, S., Lachumy, S.J., Ravichandran, M., Latha, L.Y. and Gegu, S.R. (2011) Epidemiology of Helicobacter pylori among multiracial community in Northern Peninsular, Malaysia: Effect of age across race and gender. 
Asian Pacific Journal of Tropical Medicine, 4, 72-75. http://dx.doi.org/10.1016/S1995-7645(11)60037-0

[17] Kyriazanos, I., Ilias, I., Lazaris, G., Hountis, P., Deros, I., Dafnopoulou, A. and Datsakis, K. (2001) A cohort study on Helicobacter pylori serology before and after induction in the Hellenic Navy. Military Medicine, 166, 411415.

[18] Seyda, T., Derya, C., Füsun, A. and Meliha, K. (2007) The relationship of Helicobacter pylori positivity with age, sex, and ABO/Rhesus blood groups in patients with gastrointestinal complaints in Turkey. Helicobacter, 12, 244-250. http://dx.doi.org/10.1111/j.1523-5378.2007.00500.x

[19] Kawasaki, M., Kawasaki, T., Ogaki, T., Itoh, K., Kobayashi, S., Yoshimizu, Y., Aoyagi, K., Iwakawa, A., Takahashi, S., Sharma, S. and Acharya, G.P. (1998) Seroprevalence of Helicobacter pylori infection in Nepal: Low prevalence in an isolated rural village. European Journal of Gastroenterology \& Hepatology, 10, 47-50. http://dx.doi.org/10.1097/00042737-199801000-00009

[20] Hoang, T.T., Bengtsson, C., Phung, D.C., Sörberg, M. and Granström, M. (2005) Seroprevalence of Helicobacter pylori infection in urban and rural Vietnam. Clinical and Diagnostic Laboratory Immunology, 12, 81-85. 